\section{HYGIENE AND THERAPY COURSE TURNS 30}

A dental hygiene and therapy course at Queen Mary University of London has celebrated its 30th anniversary.

Pre-1983 dental hygienists and dental therapists trained separately to dentist undergraduates. Since an integrated programme was introduced, in excess of 260 students have graduated from the dental hygiene and therapy course with an almost $100 \%$ pass rate.

\section{NEW STANDARDS PUBLISHED}

New General Dental Council (GDC) standards for the dental profession came into force on 30 September 2013. The new standards place a much stronger focus on patients' expectations and entitlements.

The standards were developed by the GDC following intensive consultation with patients and the public.

Standards for the dental team replaces Standards for dental professionals and includes nine key principles such as 'put patients' interests first' and 'raise concerns if patients are at risk'. New issues addressed in the document include an emphasis on being fluent in written and spoken English, greater emphasis on 'softer skills' and new requirements to display indicative prices for treatment.

The GDC has also updated its Scope of practice guidance to reflect recent decisions on direct access and the new standards. For dental nurses, the wording on the application of fluoride varnish has been amended to take account of direct access: for orthodontic therapists IOTN screening has been added; for dental hygienists and dental therapists the Scope now includes being able to treat patients directly, carrying out clinical examination and diagnosis within their scope of practice, and the inclusion of the care of implants and treatment of peri-implant tissues in core skills; for dental therapists direct restorations now includes secondary teeth; there is clarity that dental technicians can see patients direct to repair dentures; and there is an amendment for clinical dental technicians of providing complete dentures direct to patients to include prescribing the dentures.

The full Standards and Scope of practice documents can be found at: www.gdc-uk. org/Dentalprofessionals/Standards/Pages/ standards.aspx.

\section{IT'S ALL IN THE MIND}

How is it possible for some patients to undertake extractions and other complex procedures, without seeming to experience pain or apparent discomfort, despite not having been given any anaesthesia? What are the changes in the brain that will allow this to occur and how does hypnosis facilitate these changes?

The answer lies in the patient's perception of the experience. Pain and anxiety are inexorably linked and when patients feel anxious in treatment, their pain tolerance thresholds are reduced considerably.

Under hypnosis the part of the brain that appears to register the suffering component of pain, the anterior cingulate cortex, seems to change the way it operates and appears to recalibrate the experience in a different way. So much so that when a patient who is 'wide awake' experiences an extraction without anaesthesia this is likely to be excruciating! The patient who is hypnotised on the other hand appears to go through the experience and will report back that they were aware of the sensations of pushing, pressure and pulling but didn't feel what they would consciously label as pain in the same way as the patient who was un-anaesthetised. Furthermore it has been shown that their heart rates and blood pressure remain entirely normal and the clinician can converse and communicate with the patient during the procedure itself!

Hypnotised patients' experiences become altered as they relax and their brain states change by slowing down which allows more creative processes to take over in perception. In this altered state of awareness, the subject can begin to experience the stimulus of the clinic in other ways than that of a person who is wide awake. For example, where a patient is being told about relaxing on a beach and how nice it is, they can perceive the lights of the operating lamp above as the warm sun and the sound of the drill as passing jet aircraft in their dream like state.

Of course hypnosis isn't just restricted to practice of dentistry; it can be applied in unravelling and solving problem behaviours, phobias and additionally used as an aid for smoking cessation, weight loss, insomnia and simple relaxation too.

Anthony Asquith Dip Hyp Psych UK, the author of this article, will be running a course to introduce dental hypnosis for dental staff, in conjunction with the BDA, on Friday 31 January 2014. By the end of the course, attendees will:

- Understand what hypnosis is and the benefits of using it within clinical practice

- Understand what happens in the brain to allow hypnosis to work so well without anaesthesia

- Be able to calm highly aroused and phobic patients by normalising their fear of treatment

- Have learned how to induce trance states using simple techniques to help patients during treatment

- Understand how to use self-hypnosis for managing your own mind state.

For further information about the course, visit www.bda.org/training or call the BDA events booking line on 02075634590 .

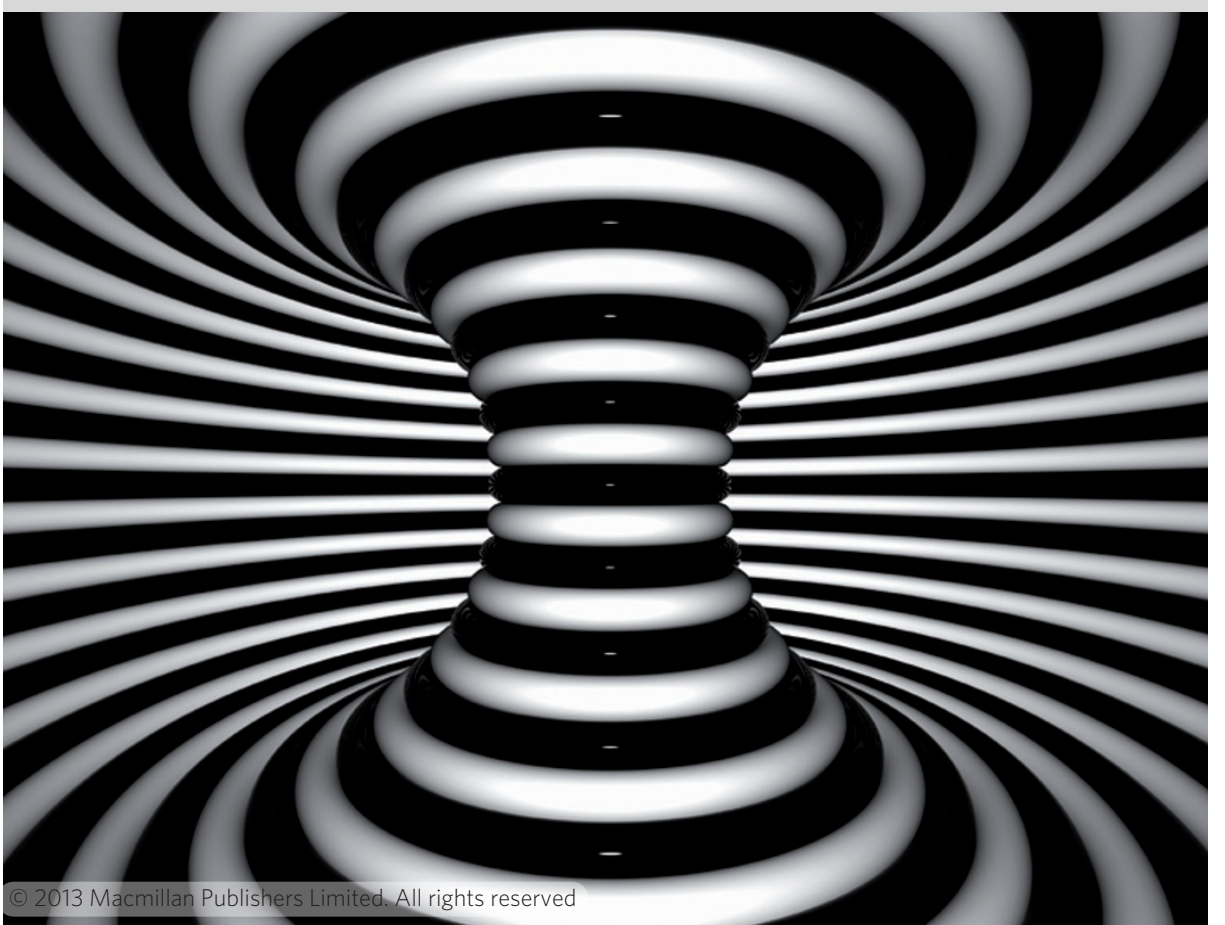

